This is an open-access article distributed under the terms of the Creative Commons Attribution License, which permits unrestricted use, distribution, and reproduction in any medium, provided the original author(s) and source are credited.

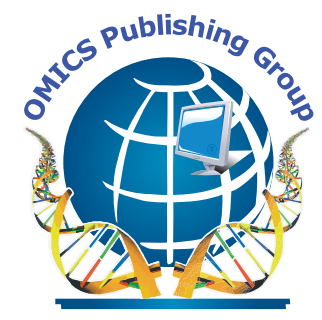

ISSN:2167-0870

\title{
Journal of Clinical Trials
}

\section{The International Open Access} Journal of Clinical Trials

\section{Executive Editors}

\section{Robert Alan Rosenheck \\ Yale University, USA}

Ulrich Rudolf Mahlknecht

Saarland University, Germany

Daniel Zelterman

Yale University, USA

Reza Hakkak

University of Arkansas for Medical Sciences, USA

Ivy M. Alexander

Yale University, USA

\section{Available online at: OMICS Publishing Group (www.omicsonline.org)}

T his article was originally published in a journal published by OMICS Publishing Group, and the attached copy is provided by OMICS Publishing Group for the author's benefit and for the benefit of the author's institution, for commercial/research/ educational use including without limitation use in instruction at your institution, sending it to specific colleagues that you know, and providing a copy to your institution's administrator.

All other uses, reproduction and distribution, including without limitation commercial reprints, selling or licensing copies or access, or posting on open internet sites, your personal or institution's website or repository, are requested to cite properly. 


\title{
A Combined Technique for Randomisation of a Small Number of Participants with a Variety of Covariates into Treatment and Control Groups in Randomised Controlled Trials
}

Roozbeh Naemi ${ }^{1},{ }^{*}$, Aoife Healy ${ }^{1}$, Lakshmi Sundar ${ }^{2,3}$, Ambadi Ramachandran $^{2}$ and Nachiappan Chockalingam ${ }^{1}$

${ }^{1}$ Staffordshire University, Stoke on Trent, Staffordshire, UK

${ }^{2}$ AR Diabetes Hospitals, Chennai, Tamil Nadu, India

${ }^{3}$ India Diabetes Research Foundation, Chennai, Tamil Nadu, India

\begin{abstract}
Background: Randomised controlled trials are widely favoured in research design as the most rigorous way of determining the effectiveness of a treatment. For assigning a small number of participants who are identified before the start of the randomisation into treatment and control, the simple randomization technique can lead to imbalance of covariates among the groups. Furthermore while the stratified randomization method can control for the effect of covariates, in smaller clinical trials, the allocation of participants to groups by flip of a coin can result in uneven arms when the number of participants in each stratum in low. Despite the ability of covariate adaptive randomization technique in minimising the difference in covariate between the arms, the techniques comes with an unnecessary increase in the computational process specifically when number of covariates increases, and when all participants are identified prior to the randomisation. The purpose of this study was to propose a method of assigning small number of participants (68) who are identified before the start of randomisation, into treatment and control arms.

Methods: The participants were first assigned into strata. For strata with even number of participants, the participants are sequentially pulled out of the strata on a random basis and assigned to arms by flip of a coin until half of the participants are assigned to any of the two arms. Then the remaining participants were assigned to the other arm. When the number of participants in a stratum is odd the first participants was pulled out of the stratum on a random basis and kept separate, then the remaining even number of participants were assigned to arms according to the method for strata that contain even number of participants. The first participants that were pulled out of the strata with odd number of participants were assigned sequentially using covariate adaptive randomisation method.
\end{abstract}

Results: Two arms were created with minimal difference between the two arms and with the sum of absolute difference equal to 12 .

Conclusions: The method showed to be able to assign small number of participants into balanced arms with minimal computational costs when a number of covariates exist.

Keywords: Randomisation; Clinical trials; Stratified randomisation; Covariate adaptive randomisation; Minimisation

\section{Introduction}

Randomised controlled trials are widely favoured in various types of research designs as the most rigorous way of determining the effectiveness of a treatment [1]. Within this research design randomization is the process through which an equal chance of being assigned to any group for which the treatment varies is given to each participant [2]. This ensures that the participants in various groups will not differ in any systematic manner.

Various study designs can detect associations between a clinical intervention and an outcome resulting from that intervention, by ruling out the possibility that the association was caused by another factor that is linked to both intervention and outcome. However, this is not a possibility where various influential factors can potentially unbalance the groups and jeopardise the results of the study. When the number of participants are more than 100 , the most simple randomization technique like flipping a coin is more likely to result in an even and homogenous groups. But for smaller clinical trials it can lead to imbalance among the groups.

To overcome the limitations of simple randomization techniques in forming homogenous groups, stratified randomization technique has been proposed that works by identifying covariates specific to the research question [3]. These factors (covariates) are used to generate a separate block for each combination of covariates, to which participants are assigned. After assigning all participants to the blocks, flip of a coin can be used to assign participants to one of the groups (arms). Despite the fact that the stratified randomization method can control for the effect of covariates when there are too many covariates to control for, there will be an increase in the number of blocks with just one participant [4]. This is specifically the case in smaller clinical trials when the number of conditions (blocks) reaches half of the sample size [5].

Furthermore in strata with odd number of participants assigning participants into arms by flip of a coin, will result in one arm having one more of the participant belonging to the specific stratum, which can affect the balance of covariates between the arms. Another method known as covariate adaptive randomization [6] has been commonly used when participants are not all identified before the start of randomisation [7]. In this method of randomisation, a new participant is assigned to a group in a sequential order through minimising the

*Corresponding author: Roozbeh Naemi, CSHER, Faculty of Health Sciences, Leek Road, Stoke on Trent ST4 2DF, UK, Tel: +44 1782 295879; Fax: +44 1782 294321; E-mail: r.naemi@staffs.ac.uk

Received August 08, 2013; Accepted December 18, 2013; Published December 20, 2013

Citation: Naemi R, Healy A, Sundar L, Ramachandran A, Chockalingam N (2013) A Combined Technique for Randomisation of a Small Number of Participants with a Variety of Covariates into Treatment and Control Groups in Randomised Controlled Trials. J Clin Trials 4: 150. doi:10.4172/2167-0870.1000150

Copyright: $\odot 2013$ Naemi R, et al. This is an open-access article distributed under the terms of the Creative Commons Attribution License, which permits unrestricted use, distribution, and reproduction in any medium, provided the original author and source are credited. 
Citation: Naemi R, Healy A, Sundar L, Ramachandran A, Chockalingam N (2013) A Combined Technique for Randomisation of a Small Number of Participants with a Variety of Covariates into Treatment and Control Groups in Randomised Controlled Trials. J Clin Trials 4: 150. doi:10.4172/2167-0870.1000150

Page 2 of 5

imbalance of several covariates between groups [8,9]. While in the study by Taves (1974) [6] the participant was assigned to the group with the lower total covariate to minimize imbalance, Pocock and Simon [10] proposed assigning participant to the group which prospectively creates less absolute differences of covariates if the participant was assigned to that group. Despite the advantages of covariate adaptive randomisation, the technique comes with an increased administrative cost due to more demand in the computational process of assignment $[7,8]$.

The purpose of this study is to propose a simple method of assigning small number of participants (less than 100) into arms when the number of strata reaches half of the number of participants and when all participants are identified before the start of randomisation process.

\section{Methods}

The ethical approval was indorsed by the ethics committee of India Diabetes Research Foundation. 68 patients were recruited after giving informed consent to participate in the study. All participants are assigned to strata based on the clinical risk factors (prognosis factor) or similar variables (covariates). For the strata with even number of participants, the first participant is pulled out of the stratum on complete random basis (i.e. by pulling the card corresponding to the participant from a bag) and is assigned by flip of a coin to one of the arms Figure 1. This is continued for the next participant in the stratum until half of the participants in this stratum are allocated to one arm. Then the remaining participants in this stratum are allocated to the other arm. This is to ensure that the likelihood of imbalance between the groups is eliminated and that the two groups get equal number of participants from the same stratum.

When the number of participants in a stratum is odd, the first participant is pulled out of the stratum on complete random basis and kept separate, and the rest of the participant are allocated to arms according to what was explained for strata with even number of participants. The first participants who were pulled out of the strata with odd number of participants (including the participant that is pulled out of the stratum with only one participant) then will be assigned, using adaptive randomisation method (Range) proposed by Pocock and Simon [10]. The diagram in Figure 1 shows the method for assignment of each participant to the arms (Figure 1).

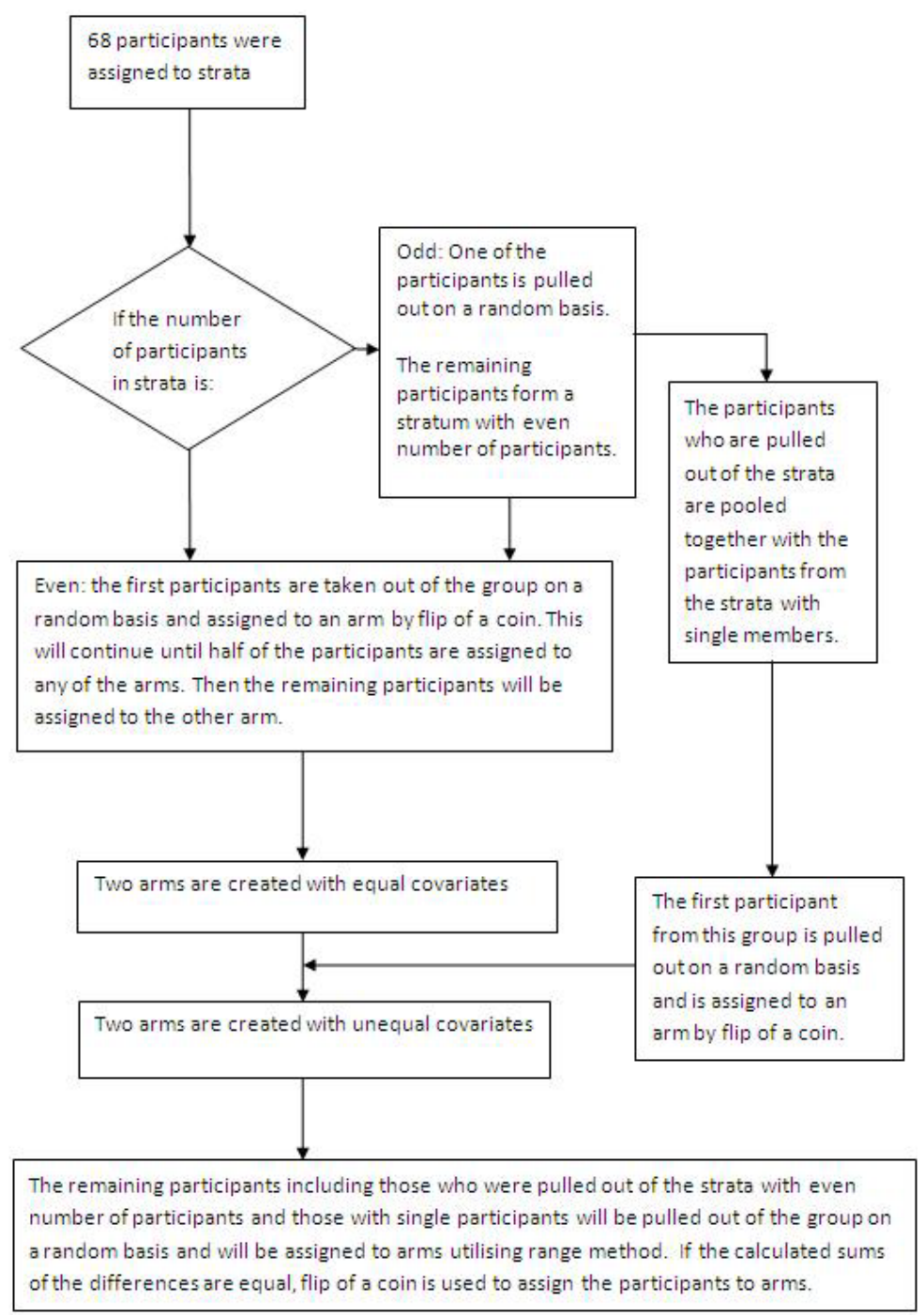

Figure 1: Method of assignment of each participant to the arms. 
Citation: Naemi R, Healy A, Sundar L, Ramachandran A, Chockalingam N (2013) A Combined Technique for Randomisation of a Small Number of Participants with a Variety of Covariates into Treatment and Control Groups in Randomised Controlled Trials. J Clin Trials 4: 150. doi:10.4172/2167-0870.1000150

Page 3 of 5

\section{A clinical example using proposed approach}

The research question within the proposed study was to identify the efficacy of using a new type of insole material in reducing the incident of foot ulceration in people with diabetes and neuropathy. In this case the plan was to assign 68 diabetic neuropathic participants; who consented to participate in a clinical trial; into 2 groups of 34 participants each, with minimal covariate (risk factors corresponding to ulceration) difference between the groups. The identified risk factors (covariates) are presented in (Table 1-3).

\section{Results}

Table 2 shows the strata and the participants in each strata, and the allocation of participants along with those who were pulled out of the strata with odd number of participants. Table 3 shows the allocation of first participants who were pulled out of the strata with odd number of participants together with the sole participants in strata with only one participant. After completing the assignment the flip of a coin was used to indicate that Arm 2 receives the new insole made of a different material. The covariate differences between the two arms are presented in Table 4.

\section{Discussion}

The combined method of assigning small number of participants into groups proposed here can be an alternative to the use of only one method of assignment like stratified and covariance adaptive randomisation. With an increased number of covariates, the method proposed here can be specifically useful when all participants are identified before assignment. In these cases there are many strata (blocks) which contain minimal participants (Weir and Lees [4]), where the use of stratified randomisation will be limited. Furthermore when the number of participants in a stratum is odd, the allocation based on flip of a coin will result in one arm having more of the participant with particular covariates that may result in imbalance of covariates between arms. On the other hand, the sequential assignment of all participant based on covariate adaptive methods $[6,10]$ comes with an increased administrative cost due to more demand in the computational process $[7,8]$. This brings unnecessary complexity when all participants are identified before the start of randomisation.

The advantage of the proposed method is that as compared to a conventional method that works based on the sequential assignment of all participants based on covariate adaptive methods $[6,10]$, only the participants from different strata which could not be assigned through simple randomisation would be assigned using covariate adaptive method. This will significantly decrease the administrative cost and computational process demand $[7,8]$ that is needed as compared to the conventional methods.

This is specifically relevant to the randomisations where all participants are identified before the start of assignment process,

\begin{tabular}{|c|c|c|c|}
\hline Risk factor & Abbreviation & 0 & 1 \\
\hline Sex & SEX & Female & Male \\
\hline Duration of diabetes (year) & DUR & $0-10$ years & Over 10 years \\
\hline Glycated Haemoglobin & $\mathrm{HbA1c}$ & Up to $7.5 \%$ & More than $7.5 \%$ \\
\hline Age (year) & Age & $18-45$ & $46-80$ \\
\hline Vibration Perception Threshold (Volt) & VPT & $25-40$ & Over 40 \\
\hline Monofilament & MFT & Loss of sensation in up to 4 sites & Loss of sensation in more than 4 sites \\
\hline Ankle Brachial Index & $\mathrm{ABI}$ & $0.9-1.2$ & Up to 0.9 or more than 1.2 \\
\hline Visual acuity & VISU & Normal & Acuity less than 6 or evidence of retinopathy \\
\hline
\end{tabular}

Table 1: The corresponding risk factors with potential contribution to ulceration.

\begin{tabular}{|c|c|c|c|c|c|c|c|c|c|c|c|c|}
\hline \multicolumn{8}{|c|}{ Strata } & \multirow[b]{2}{*}{$\begin{array}{l}\text { No of } \\
\text { Participants in } \\
\text { stratum }\end{array}$} & \multirow[b]{2}{*}{ Participants } & \multirow[b]{2}{*}{$\begin{array}{l}\text { Participants who } \\
\text { were pulled out from } \\
\text { the groups with odd } \\
\text { number of members }\end{array}$} & \multicolumn{2}{|c|}{ Assignment based on Coin flip } \\
\hline 㳮 & 岁 & $\stackrel{\frac{c}{\partial}}{\circ}$ & $\begin{array}{l}\frac{0}{⿺} \\
\text { 옴 }\end{array}$ & $\stackrel{5}{s}$ & $\stackrel{\leftarrow}{\Sigma}$ & 离 & $\stackrel{\supset}{\stackrel{D}{\supset}}$ & & & & $\stackrel{\bar{\xi}}{\frac{\varepsilon}{\alpha}}$ & $\stackrel{N}{\frac{N}{2}}$ \\
\hline M & 1 & 0 & 0 & 0 & 0 & 0 & 1 & 2 & PN3, PN4 & & PN3 & PN4 \\
\hline $\mathrm{F}$ & 1 & 0 & 1 & 0 & 0 & 0 & 0 & 2 & PN6,PN7 & & PN6 & PN7 \\
\hline M & 1 & 0 & 1 & 0 & 0 & 0 & 1 & 2 & PN15, PN9 & & PN15 & PN9 \\
\hline $\mathrm{F}$ & 1 & 0 & 0 & 1 & 1 & 0 & 0 & 2 & PN16, PN11 & & PN11 & PN16 \\
\hline M & 1 & 0 & 0 & 1 & 1 & 0 & 1 & 3 & PN31, PN22, PN33 & PN33 & PN31 & PN22 \\
\hline M & 1 & 1 & 1 & 0 & 0 & 0 & 1 & 4 & PN17, PN21, PN 23, PN 26 & & PN17, PN23 & PN21, PN26 \\
\hline M & 1 & 1 & 0 & 1 & 1 & 0 & 0 & 3 & PN25, PN19, PN27 & PN27 & PN25 & PN19 \\
\hline M & 1 & 1 & 0 & 1 & 1 & 0 & 1 & 2 & PN22, PN31 & & PN31 & PN22 \\
\hline $\mathrm{F}$ & 1 & 0 & 1 & 1 & 1 & 0 & 0 & 2 & PN30, PN24 & & PN30 & PN24 \\
\hline M & 1 & 1 & 1 & 1 & 1 & 0 & 0 & 3 & PN49,PN35, PN51 & PN51 & PN49 & PN35 \\
\hline M & 1 & 0 & 1 & 1 & 1 & 0 & 1 & 6 & PN36, PN38, PN39, PN40, PN44, PN48 & & PN36 PN40, PN48 & PN38, PN39, PN44 \\
\hline $\mathrm{F}$ & 1 & 1 & 1 & 1 & 1 & 0 & 0 & 3 & PN47, PN41, PN53 & PN53 & PN41 & PN47 \\
\hline $\mathrm{F}$ & 1 & 0 & 1 & 1 & 1 & 0 & 1 & 2 & PN43, PN42 & & PN43 & PN42 \\
\hline $\mathrm{F}$ & 1 & 1 & 0 & 1 & 1 & 0 & 1 & 2 & PN50,PN45 & & PN45 & PN50 \\
\hline M & 1 & 1 & 1 & 1 & 1 & 0 & 1 & 7 & $\begin{array}{c}\text { PN57, PN61, PN64, PN65, PN 66, } \\
\text { PN67, PN62 }\end{array}$ & PN62 & PN57, PN65, PN 66 & PN61, PN64, PN67 \\
\hline $\mathrm{F}$ & 1 & 1 & 1 & 1 & 1 & 0 & 1 & 5 & PN55, PN58, PN59, PN60, PN68 & PN68 & PN58, PN60 & PN55, PN59 \\
\hline
\end{tabular}


Citation: Naemi R, Healy A, Sundar L, Ramachandran A, Chockalingam N (2013) A Combined Technique for Randomisation of a Small Number of Participants with a Variety of Covariates into Treatment and Control Groups in Randomised Controlled Trials. J Clin Trials 4: 150. doi:10.4172/2167-0870.1000150

Page 4 of 5

\begin{tabular}{|c|c|c|c|c|c|c|c|c|c|c|c|c|}
\hline \multirow{2}{*}{$\begin{array}{c}\text { Participant } \\
\text { Code }\end{array}$} & \multicolumn{8}{|c|}{ Stratum } & \multicolumn{2}{|c|}{$\begin{array}{c}\text { The absolute difference if } \\
\text { assigned to: }\end{array}$} & \multirow[b]{2}{*}{ 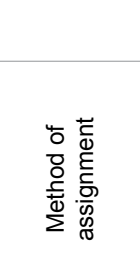 } & \multirow[b]{2}{*}{ 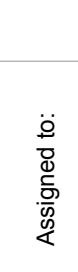 } \\
\hline & 离 & 岀 & 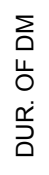 & $\begin{array}{l}\frac{0}{\dot{\alpha}} \\
\frac{0}{1}\end{array}$ & $\stackrel{\llcorner}{s}$ & $\stackrel{-\breve{L}}{\Sigma}$ & $\overline{\frac{m}{\alpha}}$ & 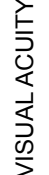 & $\bar{\xi}$ & $\underset{\mathfrak{z}}{\stackrel{N}{<}}$ & & \\
\hline PN1 & M & 1 & 0 & 0 & 0 & 0 & 0 & 1 & 0 & 0 & Coin Flip & Arm1 \\
\hline PN2 & $M$ & 1 & 0 & 0 & 0 & 0 & 0 & 0 & 16 & 4 & Cov. Adap. & Arm2 \\
\hline PN5 & $M$ & 1 & 0 & 1 & 0 & 0 & 0 & 0 & 8 & 12 & Cov. Adap. & Arm1 \\
\hline PN8 & $M$ & 0 & 0 & 1 & 0 & 0 & 0 & 1 & 16 & 4 & Cov. Adap. & Arm2 \\
\hline PN10 & $M$ & 1 & 1 & 1 & 0 & 0 & 0 & 0 & 10 & 10 & Coin Flip & Arm2 \\
\hline PN12 & $\mathrm{F}$ & 1 & 1 & 1 & 0 & 0 & 0 & 0 & 6 & 16 & Cov. Adap. & Arm1 \\
\hline PN13 & $M$ & 0 & 0 & 1 & 1 & 1 & 0 & 0 & 10 & 14 & Cov. Adap. & Arm1 \\
\hline PN14 & $\mathrm{F}$ & 1 & 1 & 0 & 0 & 0 & 0 & 1 & 18 & 12 & Cov. Adap. & Arm2 \\
\hline PN18 & $M$ & 0 & 0 & 1 & 1 & 1 & 0 & 1 & 16 & 12 & Cov. Adap. & Arm2 \\
\hline PN20 & M & 1 & 0 & 1 & 0 & 0 & 1 & 1 & 12 & 20 & Cov. Adap. & Arm1 \\
\hline PN27 & $M$ & 1 & 1 & 0 & 1 & 1 & 0 & 0 & 12 & 16 & Cov. Adap. & Arm1 \\
\hline PN28 & $\mathrm{F}$ & 1 & 1 & 1 & 0 & 0 & 0 & 1 & 18 & 14 & Cov. Adap. & Arm2 \\
\hline PN29 & $\mathrm{F}$ & 1 & 1 & 0 & 1 & 1 & 0 & 0 & 18 & 14 & Cov. Adap. & Arm2 \\
\hline PN32 & $\mathrm{F}$ & 0 & 0 & 1 & 1 & 1 & 0 & 1 & 16 & 18 & Cov. Adap. & Arm1 \\
\hline PN33 & $M$ & 1 & 0 & 0 & 1 & 1 & 0 & 1 & 18 & 18 & Coin Flip & Arm1 \\
\hline PN34 & $\mathrm{F}$ & 0 & 1 & 1 & 1 & 1 & 0 & 1 & 22 & 20 & Cov. Adap. & Arm2 \\
\hline PN37 & $M$ & 1 & 1 & 1 & 0 & 0 & 1 & 1 & 20 & 22 & Cov. Adap. & Arm1 \\
\hline PN46 & $M$ & 1 & 0 & 0 & 1 & 1 & 1 & 1 & 26 & 16 & Cov. Adap. & Arm 2 \\
\hline PN51 & $M$ & 1 & 1 & 1 & 1 & 1 & 0 & 0 & 20 & 16 & Cov. Adap. & Arm2 \\
\hline PN53 & $\mathrm{F}$ & 1 & 1 & 1 & 1 & 1 & 0 & 0 & 14 & 22 & Cov. Adap. & Arm1 \\
\hline PN56 & $M$ & 1 & 1 & 0 & 1 & 1 & 1 & 1 & 16 & 18 & Cov. Adap. & Arm1 \\
\hline PN63 & $M$ & 1 & 0 & 1 & 1 & 1 & 1 & 1 & 22 & 17 & Cov. Adap. & Arm2 \\
\hline PN68 & $\mathrm{F}$ & 1 & 1 & 1 & 1 & 1 & 0 & 1 & 12 & 16 & Cov. Adap. & Arm1 \\
\hline PN62 & M & 1 & 1 & 1 & 1 & 1 & 0 & 1 & 18 & 12 & Cov. Adap. & Arm2 \\
\hline
\end{tabular}

Table 3: Assignment of the participants from strata with single participants and those who were pulled out of the strata with odd numbers.

\begin{tabular}{|c|c|c|c|c|c|c|c|c|c|c|c|c|c|c|c|c|c|}
\hline & 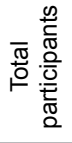 & $\frac{\frac{\omega}{N}}{\sum}$ & 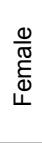 & $\begin{array}{l}\stackrel{0}{山} \\
\underset{\mathbb{2}}{0}\end{array}$ & ড্山 & $\begin{array}{l}\circ \\
\stackrel{\aleph}{\supset} \\
\text { 口 }\end{array}$ & $\begin{array}{l}\overline{\check{c}} \\
\stackrel{\underline{\Delta}}{0}\end{array}$ & $\begin{array}{l}0 \\
0 \\
\frac{1}{\delta} \\
\frac{1}{1}\end{array}$ & $\begin{array}{l}\overline{0} \\
\frac{\delta}{1} \\
\frac{O}{1}\end{array}$ & $\begin{array}{l}\circ \\
\stackrel{5}{>}\end{array}$ & $\stackrel{5}{5}$ & $\begin{array}{l}\circ \\
\frac{1}{\Sigma} \\
\frac{1}{\Sigma}\end{array}$ & $\bar{\Sigma}$ & $\frac{0}{\frac{\underline{\alpha}}{<}}$ & $\overline{\bar{\alpha}}$ & $\begin{array}{l}\stackrel{0}{\supset} \\
\stackrel{0}{>}\end{array}$ & $\underset{⿱ 亠 䒑}{\supset}$ \\
\hline ARM 1 & 34 & 21 & 13 & 24 & 10 & 16 & 18 & 10 & 24 & 10 & 24 & 10 & 24 & 31 & 3 & 12 & 22 \\
\hline ARM 2 & 34 & 22 & 12 & 25 & 9 & 15 & 19 & 10 & 24 & 10 & 24 & 10 & 24 & 32 & 2 & 10 & 24 \\
\hline $\begin{array}{l}\text { Absolute } \\
\text { difference }\end{array}$ & 0 & 1 & 1 & 1 & 1 & 1 & 1 & 0 & 0 & 0 & 0 & 0 & 0 & 1 & 1 & 2 & 2 \\
\hline
\end{tabular}

Table 4: The covariates and the sum of the absolute between the two arms. Sum of absolute difference (last row) is equal to 12.

in which utilising the conventional sequential assignment for participants from the same strata comes with unnecessary increase in processing time. This can be avoided through the utilisation of the proposed method in which rather than applying the covariate adaptive randomisation to all participants, the participant from the same strata will be assigned by simple stratified randomisation method in a much quicker way and with less processing demand. With the proposed method only one participant from strata with odd number of participants, or those in strata with single participants, will be assigned through covariate adaptive randomisation. By following this method utilising covariate adaptive randomisation method as a demanding process will be minimised while the balance between groups maintained.

The method proposed here can overcome the limitations by using the stratified and covariate adaptive randomisation in sequence. This combined setting showed to be able to produce arms with equal number of participants with minimal covariate difference between the arms. Additionally the method proposed here has specific relevance to situations where clinical trial needs to be conducted simultaneously for all participants. This can be applied to conditions in which the trial needs to be conducted in a controlled manner to avoid any chronological effect imposed by the time over which the treatment happens.

In case of randomising participants to identify the efficacy of using a new type of insole material in reducing the incident of foot ulceration, which was explained as an example here, it was decided to start the clinical trial simultaneously for all participants to avoid the effect of temperature- as a result of seasonal changes - on the insole material behaviour and its effect on increasing ulceration risk. Hence this method of randomisation that started after completion of recruitment process and before the start of the trial, showed to be effective in this case. 
Citation: Naemi R, Healy A, Sundar L, Ramachandran A, Chockalingam N (2013) A Combined Technique for Randomisation of a Small Number of Participants with a Variety of Covariates into Treatment and Control Groups in Randomised Controlled Trials. J Clin Trials 4: 150. doi:10.4172/2167-0870.1000150

Page 5 of 5

\section{Conclusion}

The purpose of this study was to assign the participant into two arms when all participants are identified before the start of assignment and when the number of covariates is high. Utilising a combination of stratified randomisation followed by an adaptive randomisation technique in sequence can provide an effective randomisation outcome.

\section{Acknowledgment}

Funding from DiabSmart project is acknowledged. Development of a new generation of 'DiaBetic' footwear using an integrated approach and Smart materials. Funded by the European Commission; Grant Agreement Number 285985, Industry Academia Partnerships and Pathways (FP7-PEOPLE-2011-IAPP).

\section{References}

1. Sibbald B, Roland M (1998) Understanding controlled trials. Why are randomised controlled trials important? BMJ 316: 201.

2. Fleiss JL, Levin B, Paik MC (2003). How to randomize. In: Statistical Methods for Rates and Proportions. ( $3^{\text {rd }}$ edn). John Wiley \& Sons pp 86-94.
3. Altman DG, Bland JM (1999) How to randomise. BMJ 319: 703-704.

4. Weir CJ, Lees KR. (2003) Comparison of stratification and adaptive methods for treatment allocation in an acute stroke clinical trial. Stat Med 22: 705-726.

5. Therneau TM (1993) How many stratification factors are "too many" to use in a randomization plan? Control Clin Trials 14: 98-108.

6. Taves DR (1974) Minimization: a new method of assigning patients to treatment and control groups. Clin Pharmacol Ther 15: 443-453.

7. Kang M, Ragan BG, Park JH (2008) Issues in outcomes research: an overview of randomization techniques for clinical trials. J Athl Train 43: 215-221.

8. Scott NW, McPherson GC, Ramsay CR, Campbell MK (2002) The method of minimization for allocation to clinical trials. a review. Control Clin Trials 23: 662 674.

9. Treasure T, MacRae KD (1998) Minimisation: the platinum standard for trials? Randomisation doesn't guarantee similarity of groups; minimisation does. BMJ 317: 362-363.

10. Pocock SJ, Simon R (1975) Sequential treatment assignment with balancing for prognostic factors in the controlled clinical trial. Biometrics 31: 103-115.
Citation: Naemi R, Healy A, Sundar L, Ramachandran A, Chockalingam N (2013) A Combined Technique for Randomisation of a Small Number of Participants with a Variety of Covariates into Treatment and Control Groups in Randomised Controlled Trials. J Clin Trials 4: 150. doi:10.4172/21670870.1000150
Submit your next manuscript and get advantages of OMICS Group submissions

Unique features:

- User friendly/feasible website-translation of your paper to 50 world's leading languages

- Audio Version of published paper

Digital articles to share and explore

Special features:

- 300 Open Access Journals

25,000 editorial team

- 21 days rapid review process

- Quality and quick editorial, review and publication processing

- Indexing at PubMed (partial), Scopus, EBSCO, Index Copernicus and Google Scholar etc

- Sharing Option: Social Networking Enabled

- Authors, Reviewers and Editors rewarded with online Scientific Credits

- Better discount for your subsequent articles

Submit your manuscript at: www.omicsonline.org/submission/ 